\title{
A two-commodity perishable inventory system
}

\author{
B Sivakuman| N Anbazhaganf G Arivarignan! \\ Received: 7 April 2005; Revised: 12 August 2005; Accepted: 18 August 2005
}

\begin{abstract}
We present a two-commodity perishable stochastic inventory system under continuous review at a service facility with a finite waiting room. The maximum storage capacity for the $i$-th item is fixed as $S_{i}(i=1,2)$. We assume that a demand for the $i$-th commodity is of unit size. The arrival instants of customers to the service station constitutes a Poisson process with parameter $\lambda$. The customer demands for these commodities are assumed to be in the ratio $p_{1}: p_{2}$. An individual customer is issued a demanded item after a random time of service with a negative exponential distribution. The items are perishable in nature and the life time of items of each commodity is assumed to be exponentially distributed. Both commodities are supposed to be substitutable in the sense that at the instant of any zero-stock, the other item may be used to meet the demand. A joint reordering policy is adopted with a random lead time for orders with exponential distribution. The joint probability distribution of the number of customers in the system and the inventory levels are obtained in both the transient and steady states. We also derive some stationary performance measures. The results are illustrated by means of a numerical example.
\end{abstract}

Key words: Stochastic inventory, two-commodity inventory, Markovian demand, service facility.

\section{Introduction}

In most of the inventory models considered in the literature, demanded items are directly delivered from stock (if available). Demand realising during stock-out periods either result in lost sales or are satisfied only after the arrival of ordered items (backlogging). In the latter case, it is assumed that either all (full backlogging) or any predetermined amount of demand (partial backlogging) realising during the stock-out period is satisfied. See Nahmias (1982), Kalpakam et al. (1990), Raafat (1991), Elango et al. (2003), Liu et al. (1999) and Yadavalli et al. (2004) for review a the above classical process.

\footnotetext{
*Department of Applied Mathematics and Statistics, Madurai Kamaraj University, Madurai, India

${ }^{\dagger}$ Department of Mathematics, Thiagarajar College of Engineering, Madurai, India

${ }^{\ddagger}$ Corresponding author: Department of Applied Mathematics and Statistics, Madurai Kamaraj University, Madurai, India, email: arivarignan@yahoo.com
} 
However, in the case of inventories maintained at service facilities, a demanded item is delivered to customers after some service time. In this case, the items are delivered not at the time of demand, but after a random time of service, causing the formation of queues. This policy necessitates a study of both the inventory level and queue length (joint) distributions.

Berman et al. (1993) considered an inventory management system at a service facility using one item of inventory for each service. They assumed that both the demand and service time are deterministic and that queues may occur only during stock-outs. They determined the optimal order quantity that minimizes the total cost rate.

Berman and Kim (1999) analyzed a problem in a stochastic environment where customers arrive at service facilities according to a Poisson process. The service times are exponentially distributed with mean inter-arrival time assumed to be larger than the mean service time. Each service requires one item from the inventory. In both the discounted case and the average cost case the optimal policy of both the finite and infinite time horizon problems is a threshold ordering policy. The optimal policy is then derived in Berman and Kim (1999), given that the order quantity is known. A logically related model has been studied by He et al. (1998), who analyzed a Markovian inventory production system, where customer demand occurs at a workshop comprising a single machine in a batch of size one. Berman and Sapna (2000) also studied an inventory control problem at a service facility requiring one item of the inventory. However, they assumed Poisson arrivals, arbitrarily distributed service times and zero lead times. They analyzed the system assuming a finite waiting room. Under a specified cost structure, they also derived the optimal ordering quantity that minimizes the long-run expected cost rate.

Elango (2001) considered a Markovian inventory system with instantaneous supply of orders at a service facility. The service time was assumed to have an exponential distribution with parameter depending on the number of waiting customers. Arivarignan et al. (2002) extended this model to include an exponential lead time. Perumal and Arivarignan ( $\overline{2002})$ considered a Markovian inventory system with an infinite waiting room. Finally, Arivarignan and Sivakumar (2003) considered an inventory system with arbitrarily distributed demand, exponential service time and exponential lead time.

As a variant, we consider a continuous review two-commodity inventory system at a service facility in this paper. For a brief review of multicommodity inventory systems we refer the reader to Goyal et al. (1989), Kalpakam et al. (1993) and Anbazhagan et al. (2000).

We also assume demand realising according to a Poisson process with fixed probabilities for the demand of both commodities which are perishable, substitutable and delivered to the customer after a random time of service. A joint reorder policy is adopted with positive random lead time. Transient and steady state analyses of the joint probability distribution for the inventory levels and the number of customers in the system are performed and various steady state performance measures are computed. Finally, the results are illustrated by means of a numerical example. 


\section{Model description}

Consider a two-commodity continuous review perishable inventory system at a service facility with a maximum capacity of $S_{i}$ units for the $i$-th commodity $(i=1,2)$. The waiting room is limited to accommodate a maximum number of $N$ customers including the one being served. The arrival instants of customers are assumed to constitute a Poisson process with parameter $\lambda(>0)$. At the starting time of service the customer demands a single item of the first commodity with probability $p_{1}$ or a single item of the second commodity with probability $p_{2}$. The demanded item is delivered to the customer after a random time of service. The service times of both commodities are assumed to be independent and exponentially distributed with parameters $\mu_{i}(i=1,2)$. The life time of each commodity is assumed to be exponentially distributed with parameter $\gamma_{i}(i=1,2)$. The reorder level for the $i$-th commodity is fixed as $s_{i}\left(1 \leq s_{i} \leq S_{i}\right)$ and an order is placed for both commodities when both the inventory levels are less than or equal to their respective reorder levels. The ordering quantity for the $i$-th commodity is $Q_{i}$ $\left(=S_{i}-s_{i}>s_{i}+1, i=1,2\right)$ items. The requirement $S_{i}-s_{i}>s_{i}+1$, ensures that after a replenishment the inventory level will always be above the respective reorder levels. The lead time is assumed to be exponentially distributed with parameter $\beta(>0)$. We also assume that the two commodities are substitutable.

The following notation is used throughout this paper:

$[A]_{i j}:(i, j)$-th element of a matrix $A$.

$A \otimes B \quad$ : Kronecker product of two matrices $A$ and $B$.

$\delta_{i j}= \begin{cases}1 & \text { if } i=j \\ 0 & \text { otherwise. }\end{cases}$

$A_{\alpha}^{*} \quad$ : Laplace transform of any arbitrary matrix $A(t)$, valid on the domain $\operatorname{Re} \alpha>0$.

0 : the zero matrix.

$\mathbf{e}^{T}:(1,1, \ldots, 1)$ - a vector containing ones.

$I$ : the identity matrix.

\section{Analysis}

Let $L_{i}(t)(i=1,2)$ denote the inventory level of the $i$-th commodity and let $X(t)$ denote the number of customers (waiting and being served) in the system, at time $t$. The stochastic process $\left\{\left(L_{1}(t), L_{2}(t), X(t)\right), t \geq 0\right\}$ has state space $E=E_{1} \times E_{2} \times E_{3}$, where $E_{1}=\left\{0,1, \ldots, S_{1}\right\}, E_{2}=\left\{0,1, \ldots, S_{2}\right\}$ and $E_{3}=\{0,1, \ldots, N\}$. From the assumptions made on the input and output processes, it may be verified that $\left(L_{1}, L_{2}, X\right) \equiv$ $\left\{\left(L_{1}(t), L_{2}(t), X(t)\right) ; t \geq 0\right\}$ with state space $E$, is a Markov process. The infinitesimal generator of this process, defined by

$$
\Theta=(\theta(i, k, m ; j, l, n)) ; \quad(i, k, m),(j, l, n) \in E,
$$

may be obtained using the following arguments:

- The arrival of a customer causes a transition from state $(i, k, m)$ to state $(i, k, m+1)$ with intensity $\lambda ; i=0,1, \ldots, S_{1} ; k=0,1, \ldots, S_{2}$ and $m=0,1, \ldots, N-1$. 
- A service completion involving the first commodity forces one customer to leave the system and a decrease of one item in the inventory level of the first commodity. Thus a transition takes place from state $(i, k, m)$ to state $(i-1, k, m-1)$ with intensity $p_{1} \mu_{1}$, where $i=1, \ldots, S_{1} ; k=1, \ldots, S_{2}$ and $m=1,2, \ldots, N$. Similarly a service completion involving the second commodity forces one customer to leave the system and a decrease of one item in the inventory level of the second commodity. Thus a transition takes place from state $(i, k, m)$ to state $(i, k-1, m-1)$ with intensity $p_{2} \mu_{2}$, where $i=1,2, \ldots, S_{1} ; k=1, \ldots, S_{2}$ and $m=1,2, \ldots, N$.

- If the inventory level of the first commodity is zero and if there is stock of the second commodity, then the demand for the first commodity is met from the stock of the second commodity and hence a transition takes place from state $(0, k, m)$ to state $(0, k-1, m-1)$ with intensity $p_{1} \mu_{2}$, where $k=1,2, \ldots, S_{2}$ and $m=1,2, \ldots, N$. Similarly if the inventory level of the second commodity is zero and if there is stock of the first commodity, then the demand for the second commodity is met from the stock of the first commodity and hence a transition takes place from state $(i, 0, m)$ to state $(i-1,0, m-1)$ with intensity $p_{2} \mu_{1}$, where $i=1,2, \ldots, S_{1}$ and $m=1,2, \ldots, N$.

- A transition from state $(i, k, m)$ to state $(i-1, k, m)$ takes place when any one of the $i$ items of the first commodity perishes at a rate $\gamma_{1}$ and the intensity for this transition is $i \gamma_{1}$, where $i=1,2, \ldots, S_{1} ; k=0,1, \ldots, S_{2}$ and $m=0,1, \ldots, N$. Similarly a transition from state $(i, k, m)$ to state $(i, k-1, m)$ takes place when any one of the $k$ items of the second commodity perishes at a rate $\gamma_{2}$ and the intensity for this transition is $k \gamma_{2}$, where $i=0,1, \ldots, S_{1} ; k=1,2, \ldots, S_{2}$ and $m=0,1, \ldots, N$.

- A transition from state $(i, k, m)$ to state $\left(i+Q_{1}, k+Q_{2}, m\right), Q_{1}=S_{1}-s_{1}, Q_{2}=S_{2}-s_{2}$, for $i=0,1, \ldots, s_{1} ; k=0,1, \ldots, s_{2}$ and $m=0,1, \ldots, N$, takes place with intensity $\beta$ when a replenishment occurs.

- For other transitions from state $(i, k, m)$ to state $(j, l, n)$, except when $(i, k, m) \neq$ $(j, l, n)$, the rate is zero.

- Finally, note that

$$
\theta(i, k, m ; i, k, m)=-\sum_{\substack{j \\(j, l, n) \neq(i, k, m)}} \sum_{l} \sum_{n} \theta(i, k, m ; j, l, n) .
$$

Hence we have

$$
\theta(i, k, m ; j, l, n)= \begin{cases}\lambda, \quad & j=i ; \quad i=0,1, \ldots, S_{1}, \\ & l=k ; \quad k=0,1, \ldots, S_{2}, \\ & n=m+1 ; \quad m=0,1, \ldots, N-1, \\ & \\ i \gamma_{1} \quad & j=i-1 ; \quad i=1,2, \ldots, S_{1}, \\ & l=k ; \quad k=0,1, \ldots, S_{2}, \\ & n=m ; \quad m=0,1, \ldots, N,\end{cases}
$$




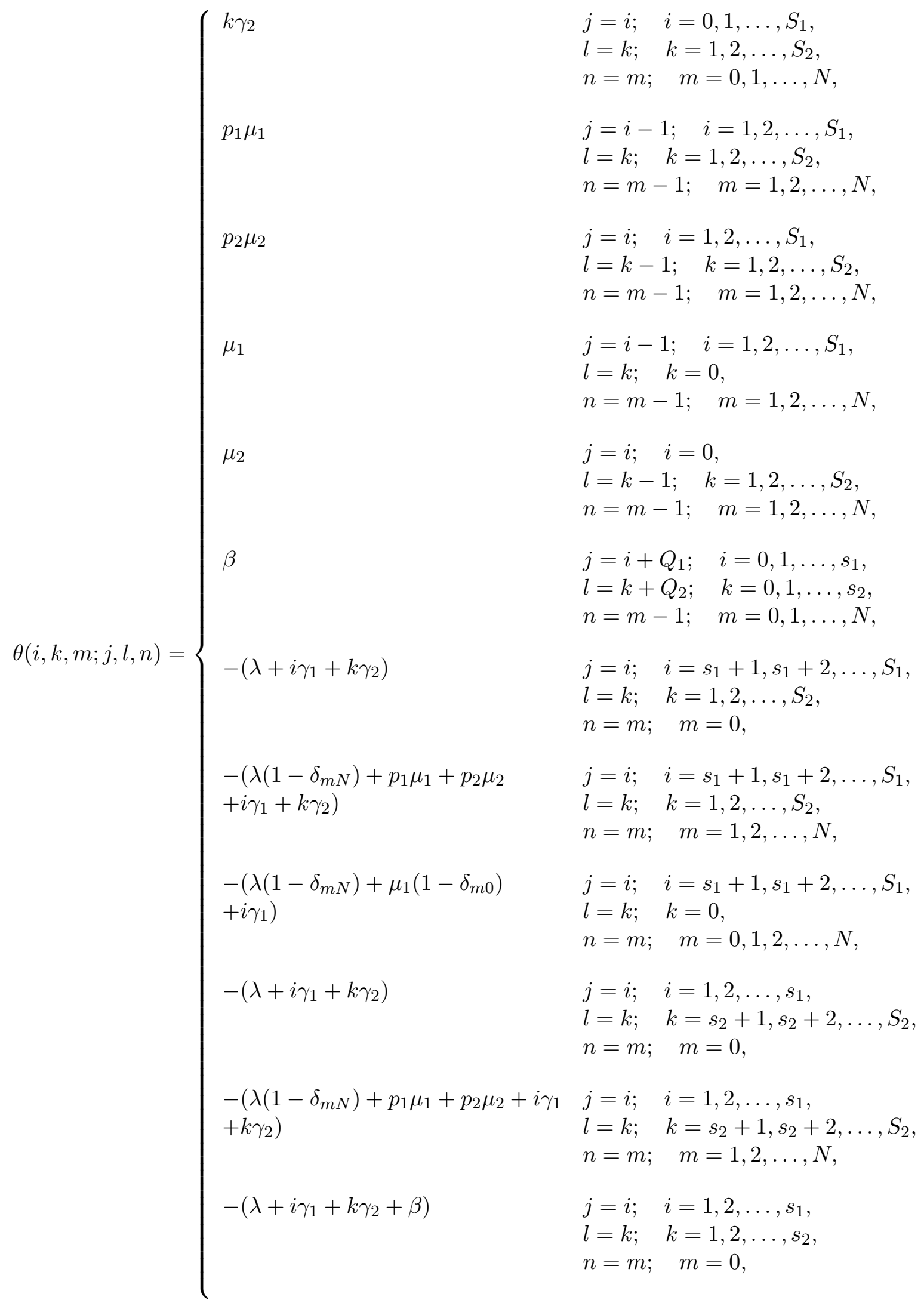


$\theta(i, k, m ; j, l, n)= \begin{cases}-\left(\lambda\left(1-\delta_{m N}\right)+p_{1} \mu_{1}+p_{2} \mu_{2}+i \gamma_{1}\right. & j=i ; \quad i=1,2, \ldots, s_{1}, \\ \left.+k \gamma_{2}+\beta\right) & l=k ; \quad k=1,2, \ldots, s_{2}, \\ & n=m ; \quad m=1,2, \ldots, N, \\ -\left(\lambda\left(1-\delta_{m N}\right)+\mu_{1}\left(1-\delta_{m 0}\right)+i \gamma_{1}+\beta\right) & j=i ; \quad i=1,2, \ldots, s_{1}, \\ & l=k ; \quad k=0, \\ & n=m ; \quad m=1,2, \ldots, N, \\ -\left(\lambda\left(1-\delta_{m N}\right)+\mu_{1}\left(1-\delta_{m 0}\right)+k \gamma_{2}\right) & j=i ; \quad i=0, \\ & l=k ; \quad k=s_{2}+1, s_{2}+2, \ldots, S_{2}, \\ & n=m ; \quad m=0,1,2, \ldots, N, \\ -\left(\lambda\left(1-\delta_{m N}\right)+\mu_{2}\left(1-\delta_{m 0}\right)+k \gamma_{2}+\beta\right) & j=i ; \quad i=0, \\ & l=k ; \quad k=1,2, \ldots, s_{2}, \\ & n=m ; \quad m=0,1, \ldots, N \\ -\left(\lambda\left(1-\delta_{m N}\right)+\beta\right) & j=i ; \quad i=0, \\ & l=k ; \quad k=0, \\ & n=m ; \quad m=0,1, \ldots, N, \\ 0 & \text { otherwise. }\end{cases}$

Let $\langle q\rangle=\left((q, 0,0),(q, 0,1), \ldots,(q, 0, N),(q, 1,0),(q, 1,1), \ldots,(q, 1, N), \ldots,\left(q, S_{2}, 0\right)\right.$, $\left.\left(q, S_{2}, 1\right), \ldots,\left(q, S_{2}, N\right)\right)$ for $q=0,1, \ldots, S_{1}$. The infinitesimal generator $\Theta$ may be expressed conveniently as a block partitioned matrix with entries

$$
[\Theta]_{i j}= \begin{cases}A_{i} & j=i ; \quad i=0,1, \ldots, S_{1} \\ B_{i} & j=i-1 ; \quad i=1,2, \ldots, S_{1} \\ C & j=i+Q_{1} ; \quad i=0,1, \ldots, s_{1} \\ \mathbf{0} & \text { otherwise. }\end{cases}
$$

More explicitly,

$\Theta=\left(\begin{array}{cccccccccccc}0 & 1 & 2 & \cdots & s_{1}-1 & s_{1} & s_{1}+1 & \cdots & S_{1}-2 & S_{1}-1 & S_{1} & \\ A_{0} & \mathbf{0} & \mathbf{0} & \cdots & \mathbf{0} & \mathbf{0} & \mathbf{0} & \cdots & \mathbf{0} & \mathbf{0} & \mathbf{0} & 0 \\ B_{1} & A_{1} & \mathbf{0} & \cdots & \mathbf{0} & \mathbf{0} & \mathbf{0} & \cdots & \mathbf{0} & \mathbf{0} & \mathbf{0} & 1 \\ \mathbf{0} & B_{2} & A_{2} & \cdots & \mathbf{0} & \mathbf{0} & \mathbf{0} & \cdots & \mathbf{0} & \mathbf{0} & \mathbf{0} & 2 \\ \vdots & \vdots & \vdots & \cdots & \vdots & \vdots & \vdots & \cdots & \vdots & \vdots & \vdots & \vdots \\ \mathbf{0} & \mathbf{0} & \mathbf{0} & \cdots & A_{s_{1}-1} & \mathbf{0} & \mathbf{0} & \cdots & \mathbf{0} & C & \mathbf{0} & s_{1}-1 \\ \mathbf{0} & \mathbf{0} & \mathbf{0} & \cdots & B_{s_{1}} & A_{s_{1}} & \mathbf{0} & \cdots & \mathbf{0} & \mathbf{0} & C & s_{1} \\ \mathbf{0} & \mathbf{0} & \mathbf{0} & \cdots & \mathbf{0} & B_{s_{1}+1} & A_{s_{1}+1} & \cdots & \mathbf{0} & \mathbf{0} & \mathbf{0} & s_{1}+1 \\ \vdots & \vdots & \vdots & \cdots & \vdots & \vdots & \vdots & \cdots & \vdots & \vdots & \vdots & \vdots \\ \mathbf{0} & \mathbf{0} & \mathbf{0} & \cdots & \mathbf{0} & \mathbf{0} & \mathbf{0} & \cdots & B_{S_{1}-1} & A_{S_{1}-1} & \mathbf{0} & S_{1}-1 \\ \mathbf{0} & \mathbf{0} & \mathbf{0} & \cdots & \mathbf{0} & \mathbf{0} & \mathbf{0} & \cdots & \mathbf{0} & B_{S_{1}} & A_{S_{1}} & S_{1}\end{array}\right.$ 
where

$$
\begin{aligned}
C= & \beta M_{1} \otimes I_{N+1}, \\
B_{i}= & I_{S_{2}+1} \otimes \tilde{B}_{i}, \quad i=1,2, \ldots, S_{1}, \\
A_{i}= & M_{2} \otimes I_{N+1}+M_{3} \otimes F_{1}+\lambda I_{S_{2}+1} \otimes F_{2}+G_{i}^{1} \otimes F_{3}+G_{i}^{2} \otimes F_{4}+G_{i}^{3} \otimes F_{5}, \\
& \quad i=s_{1}+1, s_{1}+2, \ldots, S_{1}, \\
A_{i}= & M_{2} \otimes I_{N+1}+M_{3} \otimes F_{1}+\lambda I_{S_{2}+1} \otimes F_{2}+H_{i}^{1} \otimes F_{3}+H_{i}^{2} \otimes F_{4}+H_{i}^{3} \otimes F_{5}, \\
& \quad i=1,2, \ldots, s_{1}, \\
A_{0}= & M_{2} \otimes I_{N+1}+M_{3} \otimes F_{1}+\lambda I_{S_{2}+1} \otimes F_{2}+V_{0}^{1} \otimes F_{3}+V_{0}^{2} \otimes F_{4}+V_{0}^{3} \otimes F_{5},
\end{aligned}
$$

which are expressed, in a more concise notation (as they involve a large number of zero elements) by

$$
\begin{aligned}
& {\left[M_{1}\right]_{k l}= \begin{cases}1 & l=k+Q_{2} ; k=0,1, \ldots, s_{2}, \\
0 & \text { otherwise },\end{cases} } \\
& {\left[M_{2}\right]_{k l}= \begin{cases}k \gamma_{2} & l=k-1 ; k=1,2, \ldots, S_{2}, \\
0 & \text { otherwise }\end{cases} } \\
& {\left[M_{3}\right]_{k l}= \begin{cases}1 & l=k-1 ; k=1,2, \ldots, S_{2}, \\
0 & \text { otherwise }\end{cases} }
\end{aligned}
$$

Furthermore, for $i=1,2, \ldots, S_{1}$,

$$
\begin{aligned}
& {\left[\tilde{B}_{i}\right]_{k l}= \begin{cases}i \gamma_{1} & l=k ; \quad k=0,1, \ldots, N, \\
p_{1} \mu_{1} & l=k-1 ; \quad k=1,2, \ldots, N, \\
0 & \text { otherwise, }\end{cases} } \\
& {\left[F_{1}\right]_{k l}= \begin{cases}p_{2} \mu_{2} & l=k-1 ; \quad k=1,2, \ldots, N, \\
0 & \text { otherwise, }\end{cases} } \\
& {\left[F_{2}\right]_{k l}= \begin{cases}1 & l=k+1 ; \quad k=0,1, \ldots, N-1, \\
0 & \text { otherwise },\end{cases} } \\
& {\left[F_{3}\right]_{k l}= \begin{cases}1 & l=k ; \quad k=1 ; \ldots, N-1, \\
0 & \text { otherwise },\end{cases} } \\
& {\left[F_{4}\right]_{k l}= \begin{cases}1 & l=k=0, \\
0 & \text { otherwise },\end{cases} } \\
& {\left[F_{5}\right]_{k l}= \begin{cases}1 & l=k=N, \\
0 & \text { otherwise. }\end{cases} }
\end{aligned}
$$


Also, for $i=s_{1}+1, s_{1}+2, \ldots, S_{1}$,

$$
\begin{aligned}
& {\left[G_{i}^{1}\right]_{k l}= \begin{cases}-\left(\lambda+\mu_{1}+i \gamma_{1}\right) & l=k=0, \\
-\left(\lambda+p_{1} \mu_{1}+p_{2} \mu_{2}+i \gamma_{1}+k \gamma_{2}\right) & l=k ; \quad k=1,2, \ldots, S_{2}, \\
0 & \text { otherwise, }\end{cases} } \\
& {\left[G_{i}^{2}\right]_{k l}= \begin{cases}-\left(\lambda+i \gamma_{1}\right) & l=k=0, \\
-\left(\lambda+i \gamma_{1}+k \gamma_{2}\right) & l=k ; \quad k=1,2, \ldots, S_{2}, \\
0 & \text { otherwise, }\end{cases} } \\
& {\left[G_{i}^{3}\right]_{k l}= \begin{cases}-\left(\mu_{1}+i \gamma_{1}\right) & l=k=0, \\
-\left(p_{1} \mu_{1}+p_{2} \mu_{2}+i \gamma_{1}+k \gamma_{2}\right) & l=k ; \quad k=1,2, \ldots, S_{2}, \\
0 & \text { otherwise. }\end{cases} }
\end{aligned}
$$

Finally, for $i=1,2, \ldots, s_{1}$,

$$
\begin{aligned}
& {\left[H_{i}^{1}\right]_{k l}= \begin{cases}-\left(\lambda+\mu_{1}+i \gamma_{1}+\beta\right) & l=k=0, \\
-\left(\lambda+p_{1} \mu_{1}+p_{2} \mu_{2}+i \gamma_{1}+k \gamma_{2}+\beta\right) & l=k ; \quad k=1,2, \ldots, s_{2}, \\
-\left(\lambda+p_{1} \mu_{1}+p_{2} \mu_{2}+i \gamma_{1}+k \gamma_{2}\right) & l=k ; \quad k=s_{2}+1, s_{2}+2, \ldots, S_{2}, \\
0 & \text { otherwise, }\end{cases} } \\
& {\left[H_{i}^{2}\right]_{k l}= \begin{cases}-\left(\lambda+i \gamma_{1}+\beta\right) & l=k=0 \\
-\left(\lambda+i \gamma_{1}+k \gamma_{2}+\beta\right) & l=k ; \quad k=1,2, \ldots, s_{2} \\
-\left(\lambda+i \gamma_{1}+k \gamma_{2}\right) & l=k ; \quad k=s_{2}+1, s_{2}+2, \ldots, S_{2} \\
0 & \text { otherwise, }\end{cases} } \\
& {\left[H_{i}^{3}\right]_{k l}= \begin{cases}-\left(\mu_{1}+i \gamma_{1}+\beta\right) & l=k=0 \\
-\left(p_{1} \mu_{1}+p_{2} \mu_{2}+i \gamma_{1}+k \gamma_{2}+\beta\right) & l=k ; \quad k=1,2, \ldots, s_{2} \\
-\left(p_{1} \mu_{1}+p_{2} \mu_{2}+i \gamma_{1}+k \gamma_{2}\right) & l=k ; \quad k=s_{2}+1, s_{2}+2, \ldots, S_{2} \\
0 & \text { otherwise }\end{cases} } \\
& {\left[V_{0}^{1}\right]_{k l}= \begin{cases}-(\lambda+\beta) & l=k=0 \\
-\left(\lambda+\mu_{2}+k \gamma_{2}+\beta\right) & l=k ; \quad k=1,2, \ldots, s_{2}, \\
-\left(\lambda+\mu_{2}+k \gamma_{2}\right) & l=k ; \quad k=s_{2}+1, s_{2}+2, \ldots, S_{2} \\
0 & \text { otherwise }\end{cases} } \\
& {\left[V_{0}^{2}\right]_{k l}= \begin{cases}-(\lambda+\beta) & l=k=0 \\
-\left(\lambda+k \gamma_{2}+\beta\right) & l=k ; \quad k=1,2, \ldots, s_{2}, \\
-\left(\lambda+k \gamma_{2}\right) & l=k ; \quad k=s_{2}+1, s_{2}+2, \ldots, S_{2} \\
0 & \text { otherwise, }\end{cases} } \\
& {\left[V_{0}^{3}\right]_{k l}= \begin{cases}-\beta & l=k=0 \\
-\left(\mu_{2}+k \gamma_{2}+\beta\right) & l=k ; \quad k=1,2, \ldots, s_{2}, \\
-\left(\mu_{2}+k \gamma_{2}\right) & l=k ; \quad k=s_{2}+1, s_{2}+2, \ldots, S_{2} \\
0 & \text { otherwise. }\end{cases} }
\end{aligned}
$$


It should be noted that the matrices $M, G, H$ and $V$ are square matrices of order $S_{2}+1$, whilst the matrices $\tilde{B}$ and $F$ are square matrices of order $N+1$.

\subsection{Transient analysis}

Let us define

$\psi(i, k, m ; j, l, n, t)=\operatorname{Pr}\left\{L_{1}(t)=j, L_{2}(t)=l, X(t)=n \mid L_{1}(0)=i, L_{2}(0)=k, X(0)=m\right\}$,

for all $(i, k, m),(j, l, n) \in E$. Consider the matrix $\Psi(t)=(\psi(i, k, m ; j, l, n, t))$. The Kolmogorov backward differential equation satisfied by $\Psi(t)$ is given by $\Psi^{\prime}(t)=\Psi(t) \Theta$. Clearly, $\Psi_{\alpha}^{*}=(\alpha I-\Theta)^{-1}$. The matrix $\Psi=\alpha I-\Theta$ has the block partitioned form

\begin{tabular}{|c|c|c|c|c|c|c|c|c|c|c|c|}
\hline & 0 & 1 & 2 & $\cdots$ & $s_{1}-1$ & $s_{1}$ & $s_{1}+1$ & $\cdots$ & $S_{1}-2$ & $S_{1}-1$ & $S_{1}$ \\
\hline 0 & $D_{0}$ & 0 & 0 & $\ldots$ & 0 & 0 & 0 & $\ldots$ & 0 & 0 & 0 \\
\hline 1 & $-B_{1}$ & $D_{1}$ & 0 & $\cdots$ & 0 & 0 & $\mathbf{0}$ & $\cdots$ & 0 & $\mathbf{0}$ & 0 \\
\hline 2 & 0 & $-B_{2}$ & $D_{2}$ & $\cdots$ & 0 & 0 & 0 & $\ldots$ & 0 & 0 & 0 \\
\hline & $\cdots$ & $\cdots$ & $\cdots$ & $\cdots$ & $\cdots$ & $\cdots$ & $\ldots$ & $\cdots$ & $\cdots$ & $\ldots$ & $\cdots$ \\
\hline$\vdots$ & $\cdots$ & $\ldots$ & $\cdots$ & $\ldots$ & $\cdots$ & $\ldots$ & $\ldots$ & $\ldots$ & $\cdots$ & $\ldots$ & $\cdots$ \\
\hline$s_{1}-1$ & $\mathbf{0}$ & 0 & 0 & $\cdots$ & $D_{s_{1}-1}$ & 0 & 0 & $\cdots$ & 0 & $-C$ & 0 \\
\hline$s_{1}$ & 0 & 0 & 0 & $\cdots$ & $-B_{s_{1}}$ & $D_{s_{1}}$ & 0 & $\cdots$ & 0 & 0 & $-C$ \\
\hline$s_{1}+1$ & 0 & 0 & 0 & $\cdots$ & 0 & $-B_{s_{1}+1}$ & $D_{s_{1}+1}$ & $\ldots$ & 0 & 0 & 0 \\
\hline : & $\cdots$ & $\cdots$ & $\cdots$ & $\cdots$ & $\ldots$ & $\cdots$ & $\ldots$ & $\cdots$ & $\cdots$ & $\cdots$ & $\cdots$ \\
\hline & . & $\ldots$ & $\ldots$ & $\cdots$ & $\ldots$ & $\ldots$ & $\ldots$ & $\ldots$ & . & $\ldots$ & $\ldots$ \\
\hline$S_{1}-1$ & $\mathbf{0}$ & 0 & 0 & $\cdots$ & 0 & 0 & 0 & $\cdots$ & $-B_{S_{1}-1}$ & $D_{S_{1}-1}$ & 0 \\
\hline$S_{1}$ & 0 & 0 & 0 & $\ldots$ & 0 & 0 & 0 & $\ldots$ & 0 & $-B_{S_{1}}$ & $D_{S_{1}}$ \\
\hline
\end{tabular}

where

$$
D_{i}=\alpha I-A_{i}, \quad i=0,1,2, \ldots, S_{1}
$$

It may be observed that $\alpha I-\Theta$ is an almost upper-triangular matrix in block partitioned form. That is

$$
[\Psi]_{i j}=\mathbf{0}, \quad j=0,1, \ldots, i-2 ; \quad i=2,3, \ldots, S_{1}
$$

In order to compute $\Psi^{-1}$ we proceed as follows. Consider an upper-triangular matrix $U$ and an almost upper-triangular matrix $R$ having the form

$$
U=\left(\begin{array}{ccccc}
U_{00} & U_{01} & U_{02} & \cdots & U_{0 S_{1}} \\
\mathbf{0} & U_{11} & U_{12} & \cdots & U_{1 S_{1}} \\
\mathbf{0} & \mathbf{0} & U_{22} & \cdots & U_{2 S_{1}} \\
\cdots & \cdots & \cdots & \cdots & \cdots \\
\mathbf{0} & \mathbf{0} & \mathbf{0} & \cdots & U_{S_{1} S_{1}}
\end{array}\right)
$$


with $U_{i i}=I$, for all $i=1,2, \ldots, S_{1}$, and

$$
R=\left(\begin{array}{cccccc}
R_{00} & R_{01} & R_{02} & \cdots & R_{0\left(S_{1}-1\right)} & R_{0 S_{1}} \\
-B_{1} & \mathbf{0} & \mathbf{0} & \cdots & \mathbf{0} & \mathbf{0} \\
\mathbf{0} & -B_{2} & \mathbf{0} & \cdots & \mathbf{0} & \mathbf{0} \\
\mathbf{0} & \mathbf{0} & -B_{3} & \cdots & \mathbf{0} & \mathbf{0} \\
\cdots & \cdots & \cdots & \cdots & \cdots & \cdots \\
\mathbf{0} & \mathbf{0} & \cdots & \cdots & -B_{S_{1}} & \mathbf{0}
\end{array}\right)
$$

respectively, such that $\Psi U=R$. We find the submatrices $[U]_{i j}$ and $[R]_{0 j}$ by computing the product $\Psi U$ and equating it to $R$. The $(i, j)$-th submatrix of $\Psi U$ is given by

$$
\begin{cases}-B_{i} U_{j j}, & i=1,2, \ldots, S_{1} ; j=i-1, \\
D_{i} U_{i j}-B_{i} U_{(i-1) j}, & i=s_{1}+1, \ldots, S_{1} ; j=i, i+1, \ldots, S_{1}, \\
& \text { or } \quad \begin{array}{l}
i=1,2, \ldots, s_{1} ; j=i, i+1, \ldots, Q_{1}-1, \\
D_{0} U_{0 j},
\end{array} \\
-C U_{Q_{1} j}+D_{0} U_{0 j}, & i=0 ; j=0,1,2, \ldots, Q_{1}-1, \\
-C U_{\left(Q_{1}+i\right) j}+D_{i} U_{i j}-B_{i} U_{(i-1) j}, & i=1,2, \ldots, s_{1} ; j=Q_{1}+1, Q_{1}+2 \ldots, S_{1} .\end{cases}
$$

By equating the submatrices of $\Psi U$ to the corresponding elements of $R$, we obtain

$$
[U]_{i j}= \begin{cases}\left(B_{i+1}^{-1} D_{i+1}\right)\left(B_{i+2}^{-1} D_{i+2}\right) \cdots\left(B_{j}^{-1} D_{j}\right), & i=0,1,2, \ldots, j+1 \\ & j=1,2, \ldots, Q_{1}, \\ & \text { or } \\ & i=j-Q_{1}, j-Q_{1}+1, \ldots, S_{1} \\ & j=Q_{1}+1, Q_{1}+2, \ldots, S_{1}, \\ -B_{i+1}^{-1} C U_{\left(i+Q_{1}+1\right) j}+B_{i+1}^{-1} D_{i+1} U_{(i+1) j}, & i=0,1, \ldots, j-Q_{1}-1 ; \\ & j=Q_{1}+1, Q_{1}+2, \ldots, S_{1}\end{cases}
$$

and

$$
[R]_{0 j}= \begin{cases}D_{0}, & j=0, \\ D_{0} U_{0 j}, & j=1,2, \ldots, Q_{1}-1, \\ C U_{Q_{1 j}}+D_{0} U_{0 j}, & j=Q_{1}, Q_{1}+1, \ldots, S_{1} .\end{cases}
$$

It should be noted that $B_{\alpha}^{-1}=\left(I \otimes \tilde{B}_{\alpha}\right)^{-1}=I \otimes \tilde{B}_{\alpha}^{-1}$ exists, since $\tilde{B}_{\alpha}$ is a lower-triangular matrix with zero elements, except in the main and lower diagonals.

The equation $\Psi U=R$ implies that $(\Psi U)^{-1}=R^{-1}$. Hence $U^{-1} \Psi^{-1}=R^{-1}$ and $\Psi^{-1}=$ $U R^{-1}$. It may be verified that the inverse of $R$ is given by

$$
R^{-1}=\left(\begin{array}{cccccc}
\mathbf{0} & -B_{1}^{-1} & \mathbf{0} & \cdots & \mathbf{0} & \mathbf{0} \\
\mathbf{0} & \mathbf{0} & -B_{2}^{-1} & \cdots & \mathbf{0} & \mathbf{0} \\
\cdots & \cdots & \cdots & \cdots & \cdots & \cdots \\
\cdots & \cdots & \cdots & \cdots & \cdots & \cdots \\
\mathbf{0} & \mathbf{0} & \mathbf{0} & \cdots & \mathbf{0} & -B_{S_{1}}^{-1} \\
R_{0 S_{1}}^{-1} & R_{0 S_{1}}^{-1} R_{00} B_{1}^{-1} & R_{0 S_{1}}^{-1} R_{01} B_{2}^{-1} & \cdots & R_{0 S_{1}}^{-1} R_{0\left(S_{1}-2\right)} B_{S_{1}-1}^{-1} & R_{0 S_{1}}^{-1} R_{0\left(S_{1}-1\right)} B_{S_{1}}
\end{array}\right) .
$$

From $\Psi U=R$, we obtain $\operatorname{det}(\Psi U)=\operatorname{det}(R)$ and

$$
\operatorname{det}(\Psi) \operatorname{det}(U)=\operatorname{det}\left(R_{0 S_{1}}\right) \operatorname{det}\left(-B_{1}\right) \operatorname{det}\left(-B_{2}\right) \cdots \operatorname{det}\left(-B_{S_{1}}\right) .
$$


Since $U$ is an upper-triangular matrix and the $B_{i}$ 's are lower-triangular matrices, their determinants are nonzero. Hence $\operatorname{det}\left(R_{0 S_{1}}\right) \neq 0$. This establishes the existence of the inverse $R_{0 S_{1}}^{-1}$. From $\Psi^{-1}=U R^{-1}$, we compute the $(i, j)$-th submatrix (denoted by $\Psi^{i j}$ ) of $\Psi^{-1}=(\alpha I-\Theta)^{-1}$. We obtain

$$
\Psi^{i j}= \begin{cases}U_{i S_{1}} R_{0 S_{1}}^{-1} R_{0(j-1)} B_{j}^{-1} & i=j, j+1, \ldots, S_{1} ; \quad j=1,2, \ldots, S_{1}, \\ U_{i S_{1}} R_{0 S_{1}}^{-1} & i=0,1,2, \ldots, S_{1} ; \quad j=0, \\ U_{i S_{1}} R_{0 S_{1}}^{-1} R_{0(j-1)}-U_{i(j-1)} B_{j}^{-1} & i=0,1, \ldots, S_{1}-1 ; j=i+1, \ldots, S_{1} .\end{cases}
$$

\subsection{Steady state analysis}

The structure of $\Theta$ implies that the homogeneous Markov process $\left\{\left(L_{1}(t), L_{2}(t), X(t)\right), t \geq\right.$ $0\}$ is irreducible. Hence the limiting distribution $\Phi=\left(\phi^{\mathbf{0}}, \phi^{\mathbf{1}}, \phi^{\mathbf{2}}, \ldots, \phi^{\boldsymbol{S}_{\mathbf{1}}-\mathbf{1}}, \phi^{\boldsymbol{S}_{\mathbf{1}}}\right)$, where $\phi^{m}=\left(\phi^{(m, 0,0)}, \phi^{(m, 0,1)}, \ldots, \phi^{(m, 0, N)}, \phi^{(m, 1,0)}, \phi^{(m, 1,1)}, \ldots, \phi^{(m, 1, N)}, \ldots, \phi^{\left(m, S_{2}, 0\right)}\right.$, $\left.\phi^{\left(m, S_{2}, 1\right)}, \ldots, \phi^{\left(m, S_{2}, N\right)}\right)$ for all $m=0,1, \ldots, S_{1}$ and where $\phi^{(i, k, l)}$ denotes the steady state probability of state $(i, k, l)$, exists and is given by

$$
\mathbf{\Phi} \Theta=\mathbf{0}, \quad \sum \sum_{(i, k, m)} \sum \phi^{(i, k, m)}=1 .
$$

Hence,

$$
\begin{aligned}
& \boldsymbol{\phi}^{i} B_{i}+\boldsymbol{\phi}^{\boldsymbol{i - 1}} A_{i-1}=\mathbf{0}, \quad i=1,2, \ldots, Q_{1}, \\
& \boldsymbol{\phi}^{i} B_{i}+\boldsymbol{\phi}^{i-1} A_{i-1}+\boldsymbol{\phi}^{i-1-Q_{1}} C=\mathbf{0}, \quad i=Q_{1}+1, \ldots, S_{1} \text {, } \\
& \phi^{S_{1}} A_{S}+\phi^{s_{1}} C=\mathbf{0} \text {. }
\end{aligned}
$$

After some rather tedious algebraic manipulation, we obtain

$$
\boldsymbol{\phi}^{\boldsymbol{i}}=\left\{\begin{array}{l}
(-1)^{i} \boldsymbol{\phi}^{\mathbf{0}} A_{0} B_{1}^{-1} A_{1} B_{2}^{-1} \cdots A_{i-1} B_{i}^{-1}, \quad i=1,2, \ldots, Q_{1}, \\
(-1)^{Q_{1}+1} \boldsymbol{\phi}^{\mathbf{0}} A_{0} B_{1}^{-1} A_{1} B_{2}^{-1} \cdots A_{Q_{1}} B_{Q_{1}+1}^{-1}-\boldsymbol{\phi}^{\mathbf{0}} C B_{Q_{1}+1}^{-1}, \\
(-1)^{i} \boldsymbol{\phi}^{\mathbf{0}} A_{0} B_{1}^{-1} A_{1} B_{2}^{-1} \cdots A_{i-1} B_{i}^{-1} \\
+(-1)^{i-Q_{1}} \boldsymbol{\phi}^{\mathbf{0}} C B_{Q_{1}+1}^{-1} A_{Q_{1}+1} \cdots A_{i-1} B_{i}^{-1} \\
+(-1)^{i-Q_{1}} \boldsymbol{\phi}^{\mathbf{0}}\left\{\sum_{l=1}^{i-Q_{1}-1}\left(A_{0} B_{1}^{-1} A_{1} B_{2}^{-1} \cdots A_{l-1} B_{l}^{-1}\right) C\right. \\
\left.\times\left(B_{Q_{1}+l+1}^{-1} A_{Q_{1}+l+1} \cdots B_{i}^{-1}\right)\right\}, \quad i=Q_{1}+2, Q_{1}+3, \ldots, S_{1},
\end{array}\right.
$$

where $\phi^{0}$ is determined by $\phi^{S_{1}} A_{S_{1}}+\phi^{s_{1}} C=0$ and $\sum_{i=0}^{S_{1}} \phi^{i} e=1$, i.e.

$$
\begin{aligned}
& \phi^{0}\left[(-1)^{S_{1}} A_{0} B_{1}^{-1} A_{1} B_{2}^{-1} \cdots A_{S_{1}-1} B_{S_{1}}^{-1} A_{S_{1}}+(-1)^{s_{1}} C B_{Q_{1}+1}^{-1} A_{Q_{1}+1} \cdots A_{S_{1}-1} B_{S_{1}}^{-1} A_{S_{1}}\right. \\
& +(-1)^{s_{1}}\left\{\sum_{l=1}^{s_{1}-1}\left(A_{0} B_{1}^{-1} A_{1} B_{2}^{-1} \cdots A_{l-1} B_{l}^{-1}\right) C\left(B_{Q_{1}+l+1}^{-1} A_{Q_{1}+l+1} \cdots B_{S_{1}}^{-1}\right)\right\} A_{S_{1}} \\
& \left.+(-1)^{s_{1}} A_{0} B_{1}^{-1} A_{1} B_{2}^{-1} \cdots A_{s_{1}-1} B_{s_{1}}^{-1} C\right]=0
\end{aligned}
$$


and

$$
\begin{aligned}
& \boldsymbol{\phi}^{\mathbf{0}}\left[I+\sum_{i=1}^{Q_{1}}(-1)^{i} A_{0} B_{1}^{-1} A_{1} B_{2}^{-1} \cdots A_{i-1} B_{i}^{-1}+(-1)^{Q_{1}+1} A_{0} B_{1}^{-1} A_{1} B_{2}^{-1} \cdots A_{Q_{1}} B_{Q_{1}+1}^{-1}\right. \\
& -C B_{Q_{1}+1}^{-1}+\sum_{i=Q_{1}+2}^{S 1}\left\{(-1)^{i} A_{0} B_{1}^{-1} A_{1} B_{2}^{-1} \cdots A_{i-1} B_{i}^{-1}+(-1)^{i-Q_{1}} C B_{Q_{1}+1}^{-1} A_{Q_{1}+1} \cdots A_{i-1} B_{i}^{-1}\right. \\
& \left.\left.+(-1)^{i-Q_{1}}\left\{\sum_{l=1}^{i-Q_{1}-1}\left(A_{0} B_{1}^{-1} A_{1} B_{2}^{-1} \cdots A_{l-1} B_{l}^{-1}\right) C\left[B_{Q_{1}+l+1}^{-1} A_{Q_{1}+l+1} \cdots B_{i}^{-1}\right]\right\}\right\}\right] \mathbf{e}=1 .
\end{aligned}
$$

\section{System performance measures}

In this section we derive some stationary performance measures of the system.

\subsection{Mean inventory level}

The average inventory level of the first commodity is given by

$$
\beta_{I_{1}}=\sum_{i=1}^{S_{1}} i\left(\sum_{k=0}^{S_{2}} \sum_{m=0}^{N} \phi^{(i, k, m)}\right),
$$

while that of the second commodity is

$$
\beta_{I_{2}}=\sum_{k=1}^{S_{2}} k\left(\sum_{i=0}^{S_{1}} \sum_{m=0}^{N} \phi^{(i, k, m)}\right) .
$$

\subsection{Mean reorder rate}

Let $\beta_{R}$ denote the mean reorder rate. Then we have

$$
\begin{aligned}
\beta_{R}= & p_{1} \mu_{1} \sum_{k=1}^{s_{2}} \sum_{m=1}^{N} \phi^{\left(s_{1}+1, k, m\right)}+p_{2} \mu_{2} \sum_{i=1}^{s_{1}} \sum_{m=1}^{N} \phi^{\left(i, s_{2}+1, m\right)} \\
& +\left(s_{1}+1\right) \gamma_{1} \sum_{k=0}^{s_{2}} \sum_{m=0}^{N} \phi^{\left(s_{1}+1, k, m\right)}+\left(s_{2}+1\right) \gamma_{2} \sum_{i=0}^{s_{1}} \sum_{m=0}^{N} \phi^{\left(i, s_{2}+1, m\right)} \\
& +\mu_{1} \sum_{m=1}^{N} \phi^{\left(s_{1}+1,0, m\right)}+\mu_{2} \sum_{m=1}^{N} \phi^{\left(0, s_{2}+1, m\right)}
\end{aligned}
$$

\subsection{Mean balking rate}

The mean balking rate of customers is given by

$$
\beta_{B}=\lambda \sum_{i=0}^{S_{1}} \sum_{k=0}^{S_{2}} \phi^{(i, k, N)} .
$$




\subsection{Mean perishable rate}

The mean perishable rate of the first commodity is given by

$$
\beta_{P_{1}}=\sum_{i=1}^{S_{1}} \sum_{k=0}^{S_{2}} \sum_{m=0}^{N} i \gamma_{1} \phi^{(i, k, m)},
$$

while that of the second commodity is

$$
\beta_{P_{2}}=\sum_{i=0}^{S_{1}} \sum_{k=1}^{S_{2}} \sum_{m=0}^{N} k \gamma_{2} \phi^{(i, k, m)} .
$$

\subsection{Mean waiting time}

Let $\bar{W}$ denote the mean waiting time of the customers. Then, by Little's formula (see, for example, $\operatorname{Ross}(2000))$,

$$
\bar{W}=\frac{\Gamma}{\lambda_{a}}
$$

where

$$
\Gamma=\sum_{m=1}^{N} m\left[\sum_{i=0}^{S_{1}} \sum_{k=0}^{S_{2}} \phi^{(i, k, m)}\right] .
$$

The effective arrival rate [17] is given by

$$
\lambda_{a}=\lambda \sum_{i=0}^{S_{1}} \sum_{k=0}^{S_{2}} \sum_{m=0}^{N-1} \phi^{(i, k, m)} .
$$

\subsection{Expected total cost rate}

In order to compute the total expected cost per unit time, we introduce the following notation:

$h_{1}:$ the inventory carrying cost per item per unit time of first commodity.

$h_{2}$ : the inventory carrying cost per item per unit time of second commodity.

$c_{S}:$ setup cost per order.

$c_{W}$ : waiting time cost of a customer per unit time.

$c_{P_{1}}$ : perishable cost of the first commodity per item per unit time.

$c_{P_{2}}$ : perishable cost of the second commodity per item per unit time.

$g$ : balking cost per customer per unit time.

Then the long-run expected cost rate is given by

$$
T C\left(S_{1}, S_{2}, s_{1}, s_{2}, N\right)=h_{1} \beta_{I_{1}}+h_{2} \beta_{I_{2}}+c_{S} \beta_{R}+c_{W} \bar{W}+g \beta_{B}+c_{P_{1}} \beta_{P_{1}}+c_{P_{2}} \beta_{P_{2}} .
$$

Since the computation of the $\phi^{\prime}$ s are recursive, it is quite difficult to show the convexity of the total expected cost rate. However, we present the following example to demonstrate the computability of the quantities derived in the preceding sections, and to illustrate the existence of local optima when the total cost function is treated as a function of only two variables. 


\section{Numerical illustration}

Let $S_{1}=15, S_{2}=15, N=4, \lambda=1, \mu_{1}=5, \mu_{2}=6, p_{1}=0.7, p_{2}=0.3, \gamma_{1}=0.6, \gamma_{2}=$ $0.8, \beta=0.5, h_{1}=0.2, h_{2}=0.3, c_{S}=20, c_{W}=35, g=3, c_{P_{1}}=1.5$ and $c_{P_{2}}=1$. Furthermore, let $\overline{T C}\left(s_{1}, s_{2}\right)=T C\left(15,15, s_{1}, s_{2}, 4\right)$.

\begin{tabular}{|l|l|l|l|l|l|l|l|}
\hline \multicolumn{1}{|c|}{$s_{2}$} & 1 & 2 & 3 & 4 & 5 & 6 & 7 \\
\hline 1 & 40.1443 & 39.7139 & $\mathbf{3 9 . 6 5 0 9}$ & 39.6927 & 39.7712 & 39.8743 & 40.0067 \\
2 & 39.2047 & 38.5038 & 38.3273 & $\mathbf{3 8 . 3 1 7 6}$ & 38.3575 & 38.4195 & 38.5085 \\
3 & $\underline{38.9824}$ & 38.0659 & 37.7907 & $\mathbf{3 7 . 7 4 8 0}$ & 37.7704 & 37.8051 & 37.8531 \\
4 & 39.1079 & $\underline{38.0274}$ & $\underline{37.6693}$ & $\underline{\mathbf{3 7 . 6 1 5 8}}$ & $\underline{37.6573}$ & $\underline{37.6995}$ & $\underline{37.7216}$ \\
5 & 39.4166 & 38.2100 & 37.7722 & $\mathbf{3 7 . 7 1 1 7}$ & 37.8054 & 37.9145 & 37.9597 \\
6 & 39.8350 & 38.5336 & 38.0156 & $\mathbf{3 7 . 9 2 5 7}$ & 38.0655 & 38.2902 & 38.4631 \\
7 & 40.3299 & 38.9612 & 38.3756 & $\mathbf{3 8 . 2 3 7 7}$ & 38.3782 & 38.6838 & 39.0678 \\
\hline
\end{tabular}

Table 1: Total expected cost rate.

Then the values of $\overline{T C}\left(s_{1}, s_{2}\right)$ are listed in Table 1 . The optimal cost for each $s_{1}$ is shown in bold face and the optimal cost for each $s_{2}$ is underlined. The numerical values show that $\overline{T C}\left(s_{1}, s_{2}\right)$ is a convex function in $\left(s_{1}, s_{2}\right)$ and that the optimum occurs at $\left(s_{1}, s_{2}\right)=(4,4)$. This optimum is a global optimum, since $s_{1}$ and $s_{2}$ are assumed to take all possible values $\left(\leq S_{i} / 2, i=1,2\right)$.

\section{Conclusion}

In this paper we considered a two-commodity inventory system at a service facility. This model is suitable for cases where the demanded item is delivered only after a random service time. We derived the joint probability distribution of the inventory level and the number of customers both in the transient state and in the steady state. We also derived the stationary measures of various system performances. Finally, we provided numerical evidence of the convexity of the total expected cost rate in the steady state.

\section{Acknowledgement}

The second author was supported by the DST Fast Track Scheme grant for Young Scientists and the corresponding author was supported by the National Board for Higher Mathematics, India: Research Award 48/3/2004/R\&D - II/2114. The authors wish to thank the two anonymous referees who have provided valuable suggestions for improving the presentation of this paper.

\section{References}

[1] Anbazhagan N \& Arivarignan G, 2000, Two-commodity continuous review inventory system with coordinated reorder policy, International Journal of Information and Management Sciences, 11(3), pp. 19-30. 
[2] Arivarignan G, Elango C \& Arumugam N, 2002, A continuous review perishable inventory control system at service facilities, pp. 19-40 in ARTALEJO JR \& KRISHNAmoorthy A (EDS), Advances in stochastic modelling, Notable Publications, Inc., Englewood Cliffs (NJ).

[3] Arivarignan G \& Sivakumar B, 2003, Inventory system with renewal demands at service facilities, pp. 108-123 in SRINIVAsan SK \& Vijayakumar A (EDS), Stochastic point processes, Narosa Publishing House, New Delhi.

[4] Berman O, Kaplan EH \& Shimshak DG, 1993, Deterministic approximations for inventory management at service facilities, Institute of Industial Engineers Transactions, 25, pp. 98-104.

[5] Berman O \& Kim E, 1999, Stochastic inventory policies for inventory management of service facilities, Stochastic Models, 15, pp. 695-718.

[6] Berman O \& SAPNA KP, 2000, Inventory management at service facilities for systems with arbitrarily distributed service times, Stochastic Models, 16, pp. 343-360.

[7] Elango C, 2001, A continuous review perishable inventory system at service facilities, PhD dissertation, Madurai Kamaraj University, Madurai.

[8] Elango C \& Arivarignan G, 2003, A continuous review perishable inventory systems with Poisson demand and partial backlogging, pp. 343-355 in BALAKRISHNAN N, Kannan N \& SRInivasan MR (EDs), Statistical methods and practice: Recent advances, Narosa Publishing House, New Delhi.

[9] Goyal SK \& SATIR T, 1989, Joint replenishment inventory control: Deterministic and stochastic models, European Journal of Operational Research, 38, pp. 2-13.

[10] He QM, Jewkes EM \& BuzacotT J, 1998, An efficient algorithm for computing the optimal replenishment policy for an inventory-production system, pp. 381-402, in Alfa A \& Chakravarthy S (Eds), Advances in matrix analytic methods for stochastic models, Notable Publications, Inc., Englewood Cliffs (NJ).

[11] Kalpakam S \& ARIVARIGnan G, 1990, Inventory system with random supply quantity, OR Spektrum, 12, pp. 139-145.

[12] Kalpakam S \& Arivarignan G, 1993, A coordinated multicommodity $(s, S)$ inventory system, Mathematical Computer Modelling, 18, pp. 69-73.

[13] LiU L \& YAng T, 1999, An $(s, S)$ random lifetime inventory model with a positive lead time, European Journal of Operational Research, 113, pp. 52-63.

[14] Nahmias S, 1982, Perishable inventory theory: A review, Operations Research, 30, pp. 680-708.

[15] Perumal V \& Arivarignan G, 2002, A continuous review perishable inventory system at infinite capacity service facilities, Ayya Nadar Janaki Ammal College Journal of Science, 1, pp. 37-45. 
[16] RaAfat F, 1991, A survey of literature on continuously deteriorating inventory models, Journal of the Operational Research Society, 42, pp. 27-37.

[17] Ross SM, 2000, Introduction to probability models, Harcourt Asia PTE Ltd, Singapore.

[18] Yadavalli VSS, Van Schoor CdeW, Strasheim JJ \& Udayabaskaran S, 2004, A single product perishing inventory model with demand interaction, ORiON, 20(2), pp. 109-124. 